\title{
Immunogenicity Specimen Assessments Category
}

National Cancer Institute

\section{Source}

National Cancer Institute. Immunogenicity Specimen Assessments Category. NCI

Thesaurus. Code C117557.

Classification of immunogenicity specimen assessments data. 